\title{
Overexpression of GPC3 inhibits hepatocellular carcinoma cell proliferation and invasion through induction of apoptosis
}

\author{
ZHIJIAN PAN ${ }^{*}$, CHUNZHOU CHEN*, HAOCHENG LONG ${ }^{*}$, CHANGJIANG LEI*, \\ GANG TANG, LEI LI, JIARUI FENG and FEIXIANG CHEN
}

Second Department of General Surgery, Fifth Hospital of Wuhan, Hubei 430050, P.R. China

Received October 8, 2012; Accepted January 8, 2013

DOI: $10.3892 / \mathrm{mmr} .2013 .1279$

\begin{abstract}
Glypican-3 (GPC3) is a membrane heparan sulfate proteoglycan involved in cell proliferation, differentiation, adhesion, migration and the development of the majority of mesodermal tissues and organs. GPC3 has been found to be important for the occurrence and development of hepatocellular carcinoma (HCC). Therefore, it may be suitable for use as a novel molecular marker for the diagnosis of primary liver cancer. In the present study, the role of GPC 3 in the occurrence and development of HCC was determined. GPC3 recombinant vector was transfected into two HCC cell lines, Huh7 and SK-HEP-1, to upregulate the expression of GPC3 and examine changes in the biological behavior of the cells. Results indicate that overexpression of GPC3 in Huh7 and SK-HEP-1 cells effectively inhibited cell proliferation and cell invasion through induction of apoptosis. However, cotreatment of the cells with insulin-like growth factor 2 (IGF2) and fibroblast growth factor 2 (FGF2) was found by Annexin V-PI flow cytometric analysis to significantly inhibit the apoptotic cell death induced by GPC 3 overexpression. These observations indicate that GPC3 may act as a negative regulator of IGF2 and FGF2 pathways. Taken together, these results demonstrate that overexpression of GPC 3 inhibits the occurrence and development of HCC.
\end{abstract}

\section{Introduction}

Hepatocellular carcinoma (HCC) is one of the most common malignant tumors, with morbidity and mortality of HCC ranking sixth and third among all types of cancer, respectively (1). Every year 110,000 individuals succumb to liver cancer in China, accounting for $45 \%$ of liver cancer mortalities worldwide. HCC incidence in developing countries is $\sim 5-10$ times higher

Correspondence to: Dr Zhijian Pan, Second Department of General Surgery, Fifth Hospital of Wuhan, 122 Xianzheng Street, Hanyang, Wuhan, Hubei 430050, P.R. China

E-mail: panzhijian1969@sina.com

*Contributed equally

Key words: glypican-3, hepatocellular carcinoma cells, apoptosis than in developed countries, including Europe and the United States. Epidemiological studies have identified that the rate of HCC incidence and mortality ranks second in the malignant cancers (2). HCC is characterized by a high degree of malignancy, rapid development, poor prognosis and high mortality. Surgery is currently the most effective treatment for HCC. However, metastasis and recurrence are commonly observed in $60-70 \%$ of patients following radical surgical resection. At present, there are no reliable serum markers for the diagnosis of HCC, particularly early diagnosis, thus resulting in late identification, high malignancy, rapid development, low cure rate, poor prognosis and high mortality. Therefore, the identification of highly sensitive and specific markers for predicting HCC metastasis, recurrence and prognosis is of great interest to researchers in biology, pharmacy and medicine.

Glypican-3 (GPC3) is a member of the heparan sulfate proteoglycan family of proteins and has a basic structure consisting of a core protein, a heparan sulfate chain and glycosylphosphatidylinositol (GPI). GPC3 binds to the exocytoplasmic surface of the cell membrane via the GPI anchor and regulates cell morphology, adhesion, proliferation, migration, survival and differentiation by receiving signals from receptors on the cell surface $(3,4)$. Previously, a number of studies have demonstrated that GPC 3 is important in the occurrence and development of HCC (3-7). The protein has been found to be expressed at high levels even in early-stage HCC tissues. By contrast, no expression of GPC 3 was detected in the livers of healthy adults (6). GPC3 is involved in the regulation of a number of cell physiological and pathological processes by interaction with various ligands and receptors, including cell adhesion molecules, matrix components, growth factors, enzymes and enzyme inhibitors (7). GPC3 may also be involved in inhibition and regulation of the growth of the majority of mesodermal tissues and organs, however, the underlying molecular mechanisms remain unknown. Taken together, GPC3 is of significant potential for development as a biochemical marker for diagnosis of liver cancer.

During development of HCC, GPC3 may function as a promoting factor for tumor growth (8). Studies have demonstrated that GPC3 affects HCC cell growth by crosstalk with other signaling pathways closely associated with the occurrence and development of tumors, including Wnt (8), Hedgehog (9), fibroblast growth factor (FGF) (10), insulin-like growth factor (IGF) $(11,12)$, SMAD (10) and transforming 
Table I. Primers for quantitative PCR.

\begin{tabular}{llcc}
\hline Gene & \multicolumn{1}{c}{ Primer sequences } & Product size (bp) & Accession no. \\
\hline GAPDH & F: 5'-GGTATCGTGGAAGGACTC-3' & 128 & NM_002046 \\
GPC3 & R: 5'-GTAGAGGCAGGGATGATG-3' & & \\
& F: 5'-GAGACTGCGGTGATGATGAAG-3' & 147 & NM_001164617 \\
\hline
\end{tabular}

GPC3, glypican-3.

growth factor- $\beta$ (13). However, the conclusions of previous studies are inconsistent. For example, specific studies have reported that GPC 3 promotes cancer cell growth $(8,11,13)$. By contrast, Midorikawa et al (10) demonstrated that GPC3 was overexpressed in HepG2 cells, where it interacted with FGF2 and thus inhibited cell proliferation. Kwack et al (14) also reported that forced expression of GPC3 reduced the growth of HCC cells. The inconsistencies between these studies may be explained by the diversity of various HCC cell lines. Therefore, the role of GPC3 in the development of HCC and the underlying molecular mechanisms requires further clarified. The aim of the present study was to investigate the role of GPC3 in the occurrence and development of HCC. GPC3 recombinant vector was transfected into Huh7 and SK-HEP-1 cells to upregulate the expression of GPC 3 and its biological effects on the cells. Moreover, the effects of IGF2 and FGF2 on apoptotic cell death induced by GPC 3 overexpression in HCC cells were also examined. The results indicate that overexpression of GPC 3 inhibits occurrence and development of HCC. GPC 3 may act as a negative regulator of IGF2 and FGF2 pathways.

\section{Materials and methods}

Cell culture. HCC cell lines, Huh7 and SK-HEP-1, were obtained from the China Center for Type Culture Collection (Wuhan, China) and cultured in Dulbecco's modified Eagle's medium (Gibco-BRL, Carlsbad, CA, USA) containing $10 \%$ fetal calf serum. Cells were maintained at $37^{\circ} \mathrm{C}$ in an atmosphere of humidified air with $5 \% \mathrm{CO}_{2}$ in a cell culture incubator.

Construction of GPC3 overexpression vector. RNA was extracted from HCC cells using the TRIzol Assay kit (Invitrogen Life Technologies, Carlsbad, CA, USA) according to the manufacturer's instructions, followed by reverse transcription PCR to amplify the coding region of GPC3. Then, the products were digested with $K p n I$ and $E c o$ RI (Takara Bio, Inc., Shiga, Japan), cloned into pcDNA3.1 vectors, sequenced and verified. The primers used for GPC 3 amplification were GPC 3-F (5'-cggggtaccgccaccatggccgggaccgtgcgcac-3') and GPC3-R (5'-ccggaattctcagtgcaccaggaagaagaagcacacca-3').

Transfection of plasmid. Cells were seeded into 6-well plates at $1 \times 10^{5}$ cells $/ \mathrm{ml}$ and incubated for $24 \mathrm{~h}$. Plasmid transfection was performed using Lipofectamine 2000 (Invitrogen Life Technologies) according to the manufacturer's instructions, when the cell confluence reached $\sim 70 \%$. The concentration of plasmid for transfection was $4 \mu \mathrm{g} /$ well. Following $72 \mathrm{~h}$, transfected cells were analyzed by real-time PCR and western blot analysis. For growth factor response assays, following transfection with GPC3-overexpression plasmid for $24 \mathrm{~h}$, cells were incubated in serum-free medium in the presence of $10 \mathrm{ng} / \mathrm{ml}$ FGF2 or IGF2 (R\&D Systems, Minneapolis, MN, USA) for $48 \mathrm{~h}$. Cells were then subjected to detection of cell apoptosis.

Fluorescence quantitative PCR. Total RNA was extracted from the cells using TRIzol and cDNA was prepared using $1 \mu \mathrm{g}$ RNA. Quantitative PCR was performed using SYBR-Green PCR Master Mix (Toyobo Co., Inc., Osaka, Japan), with 100 ng cDNA contained in a $20-\mu l$ reaction mixture. Primer sequences are presented in Table I. The reaction included 1 cycle of $95^{\circ} \mathrm{C}$ for $5 \mathrm{~min}$ and 40 cycles of $95^{\circ} \mathrm{C}$ for $30 \mathrm{sec}$, $55^{\circ} \mathrm{C}$ for $30 \mathrm{sec}$ and $72^{\circ} \mathrm{C}$ for $30 \mathrm{sec}$. Three independent experiments were conducted for each sample. Data were analyzed by comparing $2^{-\Delta \Delta C t}$ values.

Western blot analysis. Total cellular proteins were extracted by incubating cells in lysis buffer. Protein concentrations in the cell lysates were determined using the bicinchoninic acid assay (Pierce Biotechnology, Inc., Rockford, IL, USA). SDS-PAGE was performed on $8 \%$ glycine gels (Bio-Rad, Hercules, CA, USA), loading equal concentrations of protein per lane. Following electrophoresis, separated proteins were transferred to nitrocellulose membranes and blocked with 5\% non-fat milk in TBST buffer for $1 \mathrm{~h}$. Membranes were incubated with GPC3 and GAPDH antibodies (1:1,000; Novus Biologicals, LLC, Littleton, CO, USA) in 5\% non-fat milk overnight at $4^{\circ} \mathrm{C}$ and then anti-rabbit $\operatorname{IgG}$ monoclonal antibody conjugated with horseradish peroxidase (1:2,000; Cell Signaling Technology, Inc., Danvers, MA, USA) for $1 \mathrm{~h}$ at room temperature. Protein bands were detected using the West Femto system (Pierce Biotechnology, Inc.).

Annexin V-FLUOS apoptosis analysis. Cells were collected following transfection for $72 \mathrm{~h}$ and the translocation of phosphatidylserine in treated cells was detected using the Annexin V-FLUOS staining kit (Roche Diagnostics GmbH, Mannheim, Germany). Briefly, cells were suspended in $500 \mu \mathrm{l}$ binding buffer and incubated at room temperature in the dark for 15 min following labeling with $5 \mu \mathrm{l}$ Annexin V-fluorescein isothiocyanate (FITC) and $5 \mu 1$ propidium iodide. The stained cells were then analyzed by flow cytometry. 
A

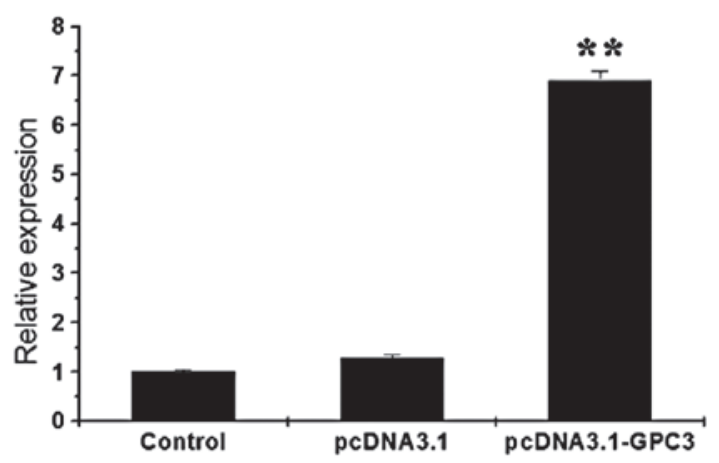

B

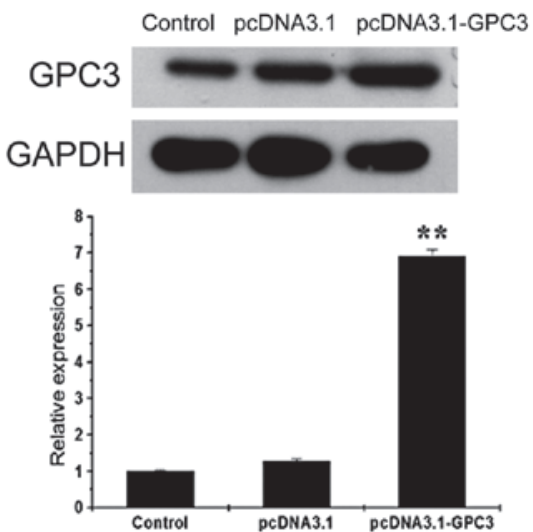

Figure 1. Overexpression of GPC3 in Huh7 cells. (A) Determination of GPC3 mRNA levels by fluorescence quantitative PCR. (B) Expression of GPC3 protein as determined by western blot analysis. Each bar represents mean \pm SD from 3 samples. GAPDH served as a loading control. ${ }^{* *} \mathrm{P}<0.05$, vs. control. GPC3, glypican-3.

EdU incorporation assay. EdU incorporation assay was performed using an EdU Apollo DNA in vitro kit (Guangzhou RiboBio Co., Ltd., Guangzhou, China) following the manufacturer's instructions. Briefly, cells were incubated with $100 \mu 1$ EdU $(50 \mu \mathrm{M}) /$ well for $2 \mathrm{~h}$. Following supernatant removal, cells were fixed for $15-30 \mathrm{~min}$ at room temperature using $100 \mu$ fixing buffer (4\% polyformaldehyde containing PBS). Following fixation, cells were incubated with $2 \mathrm{mg} / \mathrm{ml}$ glycine for 10 min followed by washing with PBS. The cells were then permeated with $100 \mu \mathrm{l}$ /well permeabilization buffer $(0.5 \%$ Triton X-100 containing PBS) and incubated with $100 \mu \mathrm{l}$ Apollo ${ }^{\circledR}$ Solution (1X) for $30 \mathrm{~min}$ at room temperature in the dark. Subsequently, incubation with $100 \mu$ l Hoechst 33342 solution (1X) was conducted for $30 \mathrm{~min}$ at room temperature in the dark. Samples were washed prior to observation under fluorescence microscopy.

Transwell Matrigel Invasion assay. Invasion of cells was evaluated by Transwell Matrigel Invasion assay. Briefly, $200 \mu \mathrm{l}$ cells following transfection $\left(1 \times 10^{6}\right.$ cells $\left./ \mathrm{ml}\right)$ and $600 \mu 1$ complete medium were added to the upper and lower compartments of the chamber, respectively. Following incubation for $48 \mathrm{~h}$, cells migrating to the lower side of the filter were fixed with $4 \%$ paraformaldehyde for $15 \mathrm{~min}$ at room temperature, washed with PBS, stained with crystal violet and then observed under a confocal microscope.

Statistical analysis. Experiments were performed in at least triplicate and results are expressed as mean \pm SD. Statistical analysis was performed using SPSS v13 (SPSS Inc., Chicago, IL, USA). $\mathrm{P}<0.05$ or $<0.01$ were considered to indicate a statistically significant difference.

\section{Results}

Overexpression of GPC3 in HCC cells. Following successful amplification of the coding region of GPC3 by PCR, the product was double digested and cloned into a pcDNA3.1 vector with $K p n \mathrm{I}$ and $E c o$ RI, followed by sequencing and verification. The GPC3 overexpression vector was transfected into Huh7 cells. Following 72 h, cells were collected and subjected

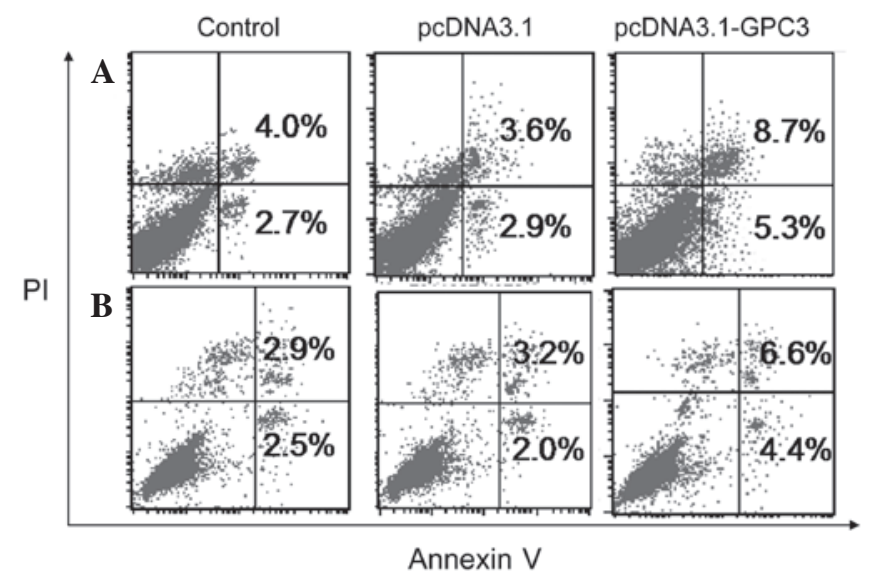

Figure 2. Effect of GPC3 on cell apoptosis as measured by Annexin V-FITC-PI co-staining flow cytometric analysis. GPC3, glypican-3; FITC, fluorescein isothiocyanate; PI, propidium iodide.

to fluorescence quantitative PCR and western blot analysis. The expression of GPC3 mRNA in Huh7 cells was found to be significantly increased following transfection, which was $\sim 7$ times that of the control group (Fig. 1). Consequently, GPC3 protein expression was also enhanced following transfection. Similarly, GPC3 in SK-HEP-1 cells at mRNA and protein levels was also increased following GPC3 overexpression (data not shown).

Effect of GPC3 on apoptosis of HCC cell lines. Resistance to apoptosis is a common characteristic of cancer cells. Therefore, the role of GPC3 in HCC development and malignance was examined by evaluating the effect of GPC3 overexpression on HCC cell apoptosis as detected by Annexin V-FLUOS flow cytometric analysis. As demonstrated in Fig. 2, overexpression of GPC3 was found to significantly induce early and late apoptosis and total apoptotic cell death in Huh7 and SK-HEP-1 cells. The different levels of apoptosis observed in the two cell lines indicated that the effects of GPC3 overexpression were cell-type specific. Taken together, the results demonstrate that overexpression of GPC 3 promotes apoptosis in HCC cells. Therefore, overexpression of GPC3 in liver cancer may inhibit the occurrence and development of HCC. 
A
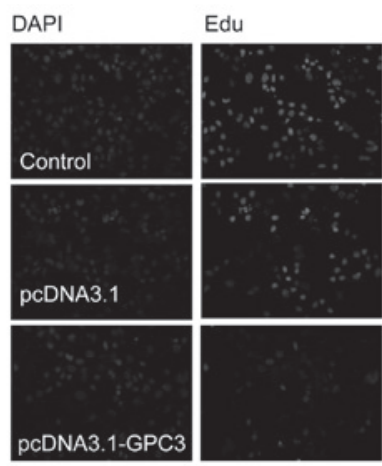

Merge

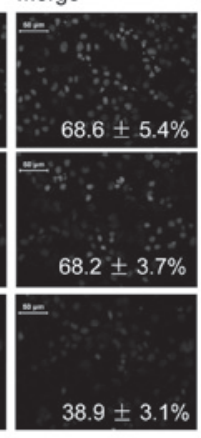

B
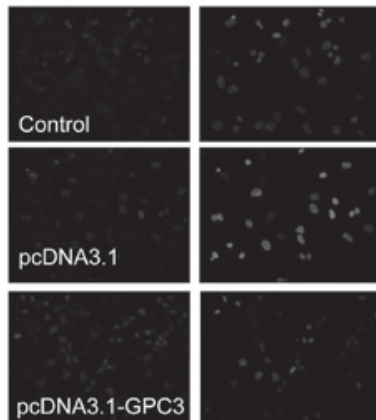

Figure 3. Effect of GPC3 overexpression on (A) Huh7 and (B) SK-HEP-1 cell proliferation. Following transfection with GPC3 overexpression or pcDNA3.1 blank vectors, cells were subjected to EdU incorporation assay as analyzed by confocal microscopy (scale bar, $50 \mu \mathrm{m}$ ). The EdU incorporation rate was expressed as the ratio of EdU positive cells to total DAPI positive cells. Results are presented as mean $\pm \mathrm{SD}(\mathrm{n}=3)$; ${ }^{*} \mathrm{P}<0.05$, vs. control. GPC3, glypican-3.
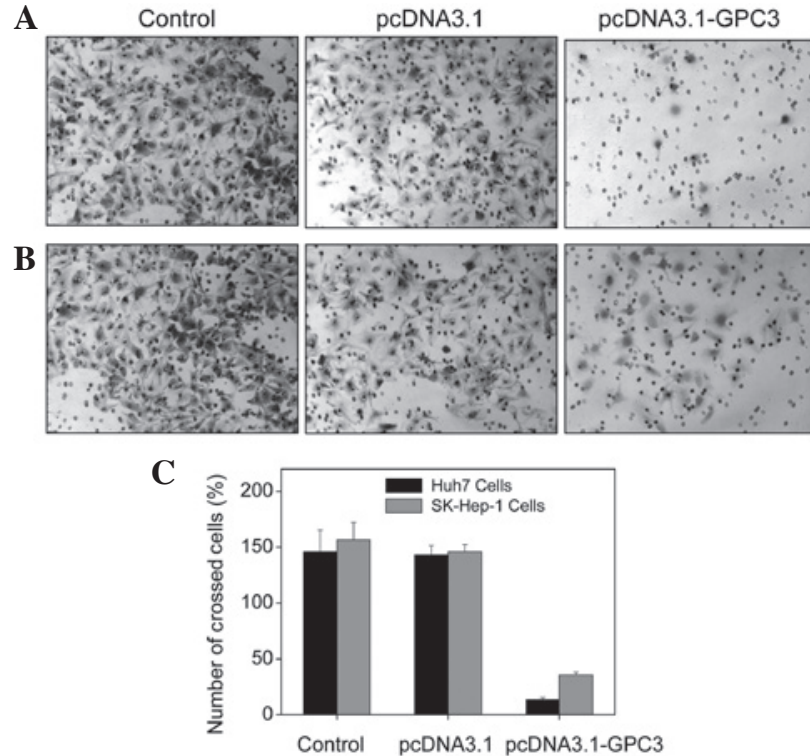

Figure 4. Effect of GPC3 overexpression on HCC cell invasion. (A) Huh7 and (B) SK-HEP-1 cells. Each bar represents mean $\pm \mathrm{SD}$ from 3 samples ${ }^{* *} \mathrm{P}<0.05$, vs. control. GPC3, glypican-3; HCC, hepatocellular carcinoma.

Role of GPC3 in HCC cell proliferation. The EdU incorporation assay, a sensitive and specific method, was employed to determine the effect of GPC3 on HCC cell proliferation. As revealed in Fig. 3A, the number of EdU-positive Huh7 cells transfected with GPC3 overexpression vectors was significantly reduced compared with those transfected with

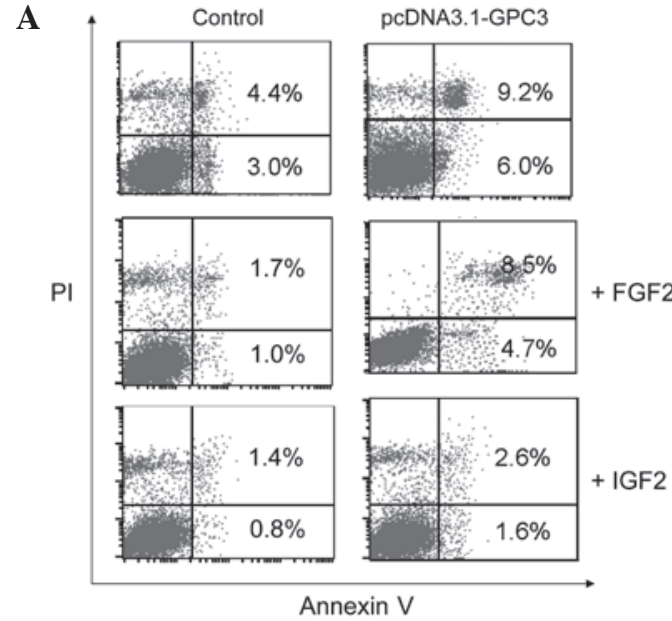

B

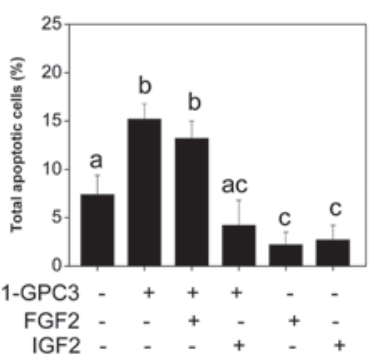

Figure 5. Effect of FGF2 or IGF2 on cell apoptosis induced by GPC3overexpression. (A) Following transfection with GPC3-overexpression plasmid for $24 \mathrm{~h}$, cells were incubated with $10 \mathrm{ng} / \mathrm{ml} \mathrm{FGF} 2$ or IGF2 for $48 \mathrm{~h}$ and cell apoptosis was analyzed by Annexin V-FITC-PI co-staining flow cytometric analysis. (B) Results are presented as mean $\pm \mathrm{SD}(\mathrm{n}=3)$. Bars with different characters $(\mathrm{a}, \mathrm{b}$ and $\mathrm{c}$ ) are statistically different at the $\mathrm{P}<0.05$ level. PC3, glypican-3; IGF insulin-like growth factor; FGF, fibroblast growth factor; FITC, fluorescein isothiocyanate; PI, propidium iodide.

pcDNA3.1 blank vector. A similar reduction at lower levels was also observed in SK-HEP-1 cells (Fig. 3B). These results indicate that overexpression of GPC3 inhibits proliferation of HCC cells.

Effect of GPC3 expression on HCC cell invasion. Malignant cancer cells may acquire the ability to invade and metastasize. In the present study, the Transwell assay was used to determine the effect of GPC3 expression on HCC cell invasion. As demonstrated in Fig. 4A, invasion of Huh7 cells was significantly inhibited following overexpression of GPC3. Similar reductions in cell invasion at lower levels were also observed in SK-HEP-1 cells (Fig. 4B). These results indicate that GPC3 is a negative regulator of $\mathrm{HCC}$ cell invasion.

Effect of IGF2 and FGF2 on HCC cell apoptosis induced by GPC 3 overexpression. To further examine the crosstalk between GPC3 and IGF2 and FGF2, growth factor response assays were conducted. Following transfection with the GPC3-overexpression plasmid, cells were subsequently incubated in serum-free medium in the presence of $10 \mathrm{ng} / \mathrm{ml} \mathrm{FGF} 2$ or IGF2 for $48 \mathrm{~h}$. Cells were then subjected to cell apoptosis detection. As shown in Fig. 5A, the early and late phase apoptosis in Huh7 cells induced by GPC 3 overexpression was inhibited by cotreatment with FGF2 and IGF2 at various levels. The antagonistic effects of IGF2 were observed to be significantly higher than that of FGF2. Results are consistent 
with the hypothesis of crosstalk between GPC3 and IGF2 and FGF2 pathways. GPC3 may act as a negative regulator of the IGF2 and FGF2 pathways.

\section{Discussion}

At present, there are no reliable serum markers for the diagnosis of HCC, particularly early diagnosis, thus resulting in late identification, high malignancy, rapid development, low cure rate, poor prognosis and high mortality. Therefore, the identification of highly sensitive and specific markers for prediction of HCC metastasis, recurrence and prognosis is an important aim of a number of biological, pharmaceutical and medical studies. Numerous studies have hypothesized that GPC3 is important in the occurrence and development of HCC and is of significant potential for development as a biochemical marker for diagnosis of liver cancer (3-7).

To determine the effects of GPC3 in HCC, the GPC3 expression vector was transfected into Huh7 and SK-HEP-1 cells and real-time PCR and western blot analysis were used to examine the changes of GPC3 at mRNA and protein levels in these cell lines. A number of previous studies have found that overexpression of GPC 3 in HepG 2 cells decreased cell apoptosis and increased cell proliferation and invasion activity. By contrast, knockdown of GPC3 resulted in converse effects $(8,11,13,15,16)$. For example, knockdown of GPC3 in MHCC97-H cells using GPC3-targeted shRNA resulted in the inhibition of cell proliferation and invasion activity (15). Sun et al (13) revealed that transfection of Huh7 and HepG2 cells with GPC3-specific siRNA effectively inhibited cell proliferation. Cheng et al (11) reported that NIH3T3 cells transfected with a GPC3 expression vector revealed overexpression of GPC3 and excessive cell proliferation. When PLC-PRF-5 HCC cells with low GPC3 expression levels were transformed into cells expressing high levels of GPC3, their growth rates were greatly increased (11). In addition, knockout of the GPC3 gene in Huh7 cells resulted in the inhibition of growth, while GPC3 activated the IGF signaling pathway and promoted growth of HCC cells (11). With respect to the underlying mechanisms through which GPC3 activated HCC cell proliferation, Capurro et al (8) indicated that GPC3 increased the signaling molecules in the classical Wnt pathway through autocrine/paracrine, while mutant GPC3 inhibited the Wnt pathway and inhibited cancer cell growth. Kittaka et al (16) demonstrated that GPC3 is an important protein in activation of the integrin signaling pathway, promoting the proliferation and invasion activity of HCC cells through associated signaling pathways.

In the present study, overexpression of GPC3 was found to result in inhibitory effects on Huh7 and SK-HEP-1 cells. Overexpression of GPC3 effectively increased cell apoptosis and inhibited cell proliferation and invasion in the cell lines. These results were consistent with previous reports $(14,17,18)$. Farooq et al (17) reported that blocking endogenous GPC3 expression with an antisense transcript promoted the growth of HepG2 and Hep3B hepatoma cells, indicating that GPC3 is an inhibitor of cell proliferation. Sung et al (18) revealed that antisense-mediated knockdown of GPC3 in HepG2 cells significantly promoted cell growth through pathways independent of IGF2. Furthermore, Kwack et al (14) demonstrated that forced expression of GPC3 effectively reduced HCC cell growth and FGF2-mediated cell proliferation was inhibited by GPC3. In addition, the authors reported that adhesion of HCC cells to collagen type I and fibronectin was decreased by GPC3, whereas cell migration and invasion were stimulated (14).

The growth inhibition of GPC3 overexpression on Huh7 and SK-HEP-1 HCC cells was similar to the action of GPC3 in healthy tissues. Previous studies have demonstrated that GPC3 is important for formation and development of tissue and organs during the embryo and fetus periods. GPC3 largely functions as a negative regulator of excessive growth of tissues and organs to regulate the overall body size (19). Liu et al (20) found that expression of GPC3 increased 2 days after hepatectomy, peaking at day 5. When expression levels of GPC3 peaked in healthy liver cells, cell division and proliferation began to decline. Therefore, it was concluded that GPC3 functioned as a negative regulator of liver regeneration and hepatocyte proliferation with the involvement of CD81. Further studies on GPC3 transgenic mice found that overexpression of GPC3 suppressed hepatocyte proliferation and liver regeneration (21). Lin et al (22) also revealed that hepatocyte-targeted overexpression of GPC3 in GPC3 trangenic mice was important for regulation of liver size and termination of hepatocyte proliferation induced by the xenobiotic mitogens phenobarbital and TCPOBOP. Mutation and loss of function of GPC3 led to excessive growth and Simpson-Golabi-Behmel syndrome (23), which is characterized by excessive growth of the liver and other organs and increased risk of cancer, particularly HCC and Wilms tumor (24). Zittermann et al (25) found that sGPC3-expressing cells exhibited a lower proliferation rate. In addition, sGPC3 was identified to significantly inhibit in vivo growth of Huh6, HepG2 and Huh7 HCC cells. Moreover, this study revealed that sGPC3 blocked Wnt signaling in Huh6and Huh7-derived tumors and Erk1/2 and Akt phosphorylation in tumors generated by Huh7 and HepG2 cells, respectively. Significant anti-angiogenic effects of GPC3 in Huh7 and HepG2-derived tumors were observed. Therefore, the authors concluded that sGPC3 inhibited HCC tumorigenicity by blocking the activity of several pro-tumorigenic growth factors. The observations of Feng et al (26) are consistent with this conclusion.

FGFs are a family of broad-spectrum growth factors affecting a plethora of cellular activities. The interaction of at least 23 ligands, 4 receptors and multiple coreceptors provides a significant complexity to a signaling system capable of effecting a multitude of responses (27). FGF2 is produced by epithelial and cancer cells and is involved in developmental processes and the regulation of differentiation, proliferation and migration. FGF2 is a critical factor for embryonic stem cell growth in culture without induction of differentiation. Signaling cascades activated through FGF2 binding to the FGF receptor include the ras-raf-MAPK, PLC $\gamma / \mathrm{PKC}$ and $\mathrm{PI} 3 \mathrm{~K} / \mathrm{AKT}$ pathways (28). IGF2 is a potent cellular mitogen primarily produced by the liver and is frequently overexpressed in tumors. IGF2 binds to the IGF-I receptor, activating the AKT, mTOR, ERK and JNK pathways (29). Aberrant levels of IGF2 are associated with Wilms tumor, Beckwith-Wiedmann syndrome and colorectal cancer (29). Crosstalk between GPC3 and FGF2 and IGF2 has also been reported in previous studies $(4,6,7,22-24,30)$. Farooq et al reported that blocking 
endogenous GPC3 expression with an antisense transcript promoted the growth of HepG2 and Hep3B cell lines (17). Sung et al also reported that antisense-mediated knockdown of GPC3 in HepG2 cells significantly promoted the growth of hepatoma cells and this growth promotion was independent of the IGF2 signaling pathway (18). In addition, Kwack et al found that FGF2-mediated cell proliferation was inhibited by GPC3 (14). However, in a breast cancer cell model, Peters et al (30) identified that GPC3 inhibited invasion and migration of the cancer cells. Additional studies in ovarian, lung, mesothelioma and breast cancer cell models also demonstrated that, overexpression of GPC3 inhibits cancer cell proliferation (31-34). Furthermore, these studies revealed that addition of IGF2 suppressed the apoptosis-inducing effects of GPC3. By contrast, FGF2 demonstrated reduced effects $(4,6,7)$. In the current study, growth factor response assays were conducted to examine the crosstalk between GPC3 and IGF2 and FGF2 in HCC cells. The results indicate that early and late phase apoptosis in Huh7 cells induced by GPC3 overexpression was inhibited by cotreatment of FGF2 and IGF2 at various levels. The antagonistic effect of IGF2 was significantly higher than that of FGF2 (Fig. 5). These results are consistent with those of previous studies, which confirmed the crosstalk between GPC3 and IGF2 and FGF2 pathways. Taken together, these observations indicate that GPC3 functions as a negative regulator of the IGF2 and FGF2 pathways.

In conclusion, HCC cells are different from human hepatoma cells, thus, the actions of GPC3 may be cell-type specific. The distinct effects of GPC3 on various HCC cells may be attributed to the varied expression levels of GPC3. These results were consistent with the hypothesis that GPC3 inhibits cell proliferation and invasion through induction of apoptosis at specific concentrations. Taken together, these results suggest that overexpression of GPC3 inhibits the occurrence and development of HCC.

\section{References}

1. El-Serag HB and Rudolph KL: Hepatocellular carcinoma: epidemiology and molecular carcinogenesis. Gastroenterology 132 2557-2576, 2007.

2. Yuen MF and Lai CL: Serological markers of liver cancer. Best Pract Res Clin Gastroenterol 19: 91-99, 2005.

3. Liu H, Li P, Zhai Y, et al: Diagnostic value of glypican-3 in serum and liver for primary hepatocellular carcinoma. World J Gastroenterol 16: 4410-4415, 2010.

4. Akutsu N, Yamamoto H, Sasaki S, et al: Association of glypican-3 expression with growth signaling molecules in hepatocellular carcinoma. World J Gastroenterol 16: 3521-3528, 2010.

5. Yan B, Wei JJ, Qian YM, et al: Expression and clinicopathologic significance of glypican 3 in hepatocellular carcinoma. Ann Diagn Pathol 15: 162-169, 2011.

6. Iglesias BV, Centeno G, Pascuccelli H, et al: Expression pattern of glypican-3 (GPC3) during human embryonic and fetal development. Histol Histopathol 23: 1333-1340, 2008.

7. Hippo Y, Watanabe K, Watanabe A, et al: Identification of soluble NH2-terminal fragment of glypican-3 as a serological marker for early-stage hepatocellular carcinoma. Cancer Res 64: 2418-2423, 2004.

8. Capurro MI, Xiang YY, Lobe C and Filmus J: Glypican-3 promotes the growth of hepatocellular carcinoma by stimulating canonical Wnt signaling. Cancer Res 65: 6245-6254, 2005.

9. Capurro MI, Xu P, Shi W, Li F, Jia A and Filmus J: Glypican-3 inhibits Hedgehog signaling during development by competing with patched for Hedgehog binding. Dev Cell 14: 700-711, 2008.
10. Midorikawa Y, Ishikawa S, Iwanari H, et al: Glypican-3, overexpressed in hepatocellular carcinoma, modulates FGF2 and BMP-7 signaling. Int J Cancer 103: 455-465, 2003.

11. Cheng W, Tseng CJ, Lin TT, et al: Glypican-3-mediated oncogenesis involves the insulin-like growth factor-signaling pathway. Carcinogenesis 29: 1319-1326, 2008.

12. Sakurai M, Shibata K, Umezu T, et al: Growth-suppressing function of glypican-3 (GPC3) via insulin like growth factor II (IGF-II) signaling pathway in ovarian clear cell carcinoma cells. Gynecol Oncol 119: 332-336, 2010.

13. Sun CK, Chua MS, He J and So SK: Suppression of glypican 3 inhibits growth of hepatocellular carcinoma cells through up-regulation of TGF- 32. Neoplasia 13: 735-747, 2011.

14. Kwack MH, Choi BY and Sung YK: Cellular changes resulting from forced expression of glypican-3 in hepatocellular carcinoma cells. Mol Cells 21: 224-228, 2006.

15. Ruan J, Liu F, Chen X, et al: Inhibition of glypican-3 expression via RNA interference influences the growth and invasive ability of the MHCC97-H human hepatocellular carcinoma cell line. Int J Mol Med 28: 497-503, 2011.

16. Kittaka N, Takemasa I, Takeda Y, et al: Molecular mapping of human hepatocellular carcinoma provides deeper biological insight from genomic data. Eur J Cancer 44: 885-897, 2008.

17. Farooq M, Hwang SY, Park MK, Kim JC, Kim MK and Sung YK: Blocking endogenous glypican-3 expression releases Hep 3B cells from G1 arrest. Mol Cells 15: 356-360, 2003.

18. Sung YK, Hwang SY, Farooq M, Kim JC and Kim MK: Growth promotion of HepG2 hepatoma cells by antisense-mediated knockdown of glypican-3 is independent of insulin-like growth factor 2 signaling. Exp Mol Med 35: 257-262, 2003.

19. Oliver F, Christians JK, Liu X, et al: Regulatory variation at glypican-3 underlies a major growth QTL in mice. PLoS Biol 3: e135, 2005.

20. Liu B, Paranjpe S, Bowen WC, et al: Investigation of the role of glypican 3 in liver regeneration and hepatocyte proliferation. Am J Pathol 175: 717-724, 2009.

21. Liu B, Bell AW, Paranjpe S, et al: Suppression of liver regeneration and hepatocyte proliferation in hepatocyte-targeted glypican 3 transgenic mice. Hepatology 52: 1060-1067, 2010.

22. Lin CW, Mars WM, Paranjpe S, et al: Hepatocyte proliferation and hepatomegaly induced by phenobarbital and 1,4-bis [2-(3,5-dichloropyridyloxy)] benzene is suppressed in hepatocyte-targeted glypican 3 transgenic mice. Hepatology 54: 620-630, 2011.

23. Pilia G, Hughes-Benzie RM, MacKenzie A, et al: Mutations in GPC3, a glypican gene, cause the Simpson-Golabi-Behmel overgrowth syndrome. Nat Genet 12: 241-247, 1996.

24. Lapunzina P: Risk of tumorigenesis in overgrowth syndromes: a comprehensive review. Am J Med Genet C Semin Med Genet 137C: 53-71, 2005.

25. Zittermann SI, Capurro MI, Shi W and Filmus J: Soluble glypican 3 inhibits the growth of hepatocellular carcinoma in vitro and in vivo. Int J Cancer 126: 1291-1301, 2010.

26. Feng M, Kim H, Phung Y and Ho M: Recombinant soluble glypican 3 protein inhibits the growth of hepatocellular carcinoma in vitro. Int J Cancer 128: 2246-2247, 2011.

27. Bansal R: Fibroblast growth factors and their receptors in oligodendrocyte development: implications for demyelination and remyelination. Dev Neurosci 24: 35-46, 2002.

28. Acevedo VD, Ittmann M and Spencer DM: Paths of FGFR-driven tumorigenesis. Cell Cycle 8: 580-588, 2009.

29. Pollak M: Insulin and insulin-like growth factor signalling in neoplasia. Nat Rev Cancer 8: 915-928, 2008.

30. Peters MG, Farías E, Colombo L, Filmus J, Puricelli L and Bal de Kier Joffé E: Inhibition of invasion and metastasis by glypican-3 in a syngeneic breast cancer model. Breast Cancer Res Treat 80: 221-232, 2003.

31. Lin H, Huber R, Schlessinger D and Morin PJ: Frequent silencing of the GPC3 gene in ovarian cancer cell lines. Cancer Res 59: 807-810, 1999 .

32. Kim H, Xu GL, Borczuk AC, et al: The heparan sulfate proteoglycan GPC3 is a potential lung tumor suppressor. Am J Respir Cell Mol Biol 29: 694-701, 2003.

33. Murthy SS, Shen T, De Rienzo A, et al: Expression of GPC3, an X-linked recessive overgrowth gene, is silenced in malignant mesothelioma. Oncogene 19: 410-416, 2000.

34. Xiang YY, Ladeda V and Filmus J: Glypican-3 expression is silenced in human breast cancer. Oncogene 20: 7408-7412, 2001. 